\title{
Direct imaging of lattice-strain-induced stripe phases in an optimally doped manganite film
}

\author{
L. Sudheendra, ${ }^{1, *, \dagger}$ V. Moshnyaga, ${ }^{1}$ E. D. Mishina, ${ }^{2}$ B. Damaschke, ${ }^{1}$ Th. Rasing, ${ }^{3}$ and K. Samwer ${ }^{1, * \frac{1}{*}}$ \\ ${ }^{1}$ Physikalisches Institut, Friedrich-Hund-Platz 1, Universitaet Goettingen, D37077 Goettingen, Germany \\ ${ }^{2}$ Moscow State Institute of Radioengineering, Electronics and Automation, Prospekt Vernadskogo 78, 119454 Moscow, Russia \\ ${ }^{3}$ Institute for Molecules and Materials, Radboud University Nijmegen, Toernooiveld 1, 6525ED Nijmegen, The Netherlands
}

(Received 6 March 2007; revised manuscript received 15 March 2007; published 31 May 2007)

\begin{abstract}
In a $\mathrm{La}_{0.75} \mathrm{Ca}_{0.25} \mathrm{MnO}_{3} / \mathrm{MgO}$ film without quenched disorder, we show insulating and metallic stripes above and below the Curie temperature $\left(T_{C}\right)$, respectively, by a high-resolution scanning tunneling microscopy (STM) and/or spectroscopy measurement. Formation of these stripes involves competing charge, orbital, and lattice orders and is an outcome of an overlapping of electron wave functions mediated by the lattice strain. The presence of the quadrupolar contribution in the second-harmonic generation experiments supports the strain mediated charge-orbital stripes observed by STM.
\end{abstract}

DOI: 10.1103/PhysRevB.75.172407

PACS number(s): 75.47.Lx, 68.37.Ef, 71.30. $+\mathrm{h}, 71.38 .-\mathrm{k}$

Stripes in manganites, ${ }^{\prime}$ like in nickelites, ${ }^{2}$ were known to comprise of ordered polarons. As the coupling of the electron to the lattice was shown to be weak, recent experiments suggest a charge-density-wave-type modulation in manganites, instead. ${ }^{3}$ In colossal magnetoresistant ${ }^{4}$ (CMR) manganites, of the form $\mathrm{La}_{1-x} \mathrm{Ca}_{x} \mathrm{MnO}_{3}(0.2 \leqslant x \leqslant 0.33)$, the localizing effect of the charge due to the Coulomb repulsion and the Jahn-Teller (JT) effect at the Mn site results in polarons. ${ }^{5,6}$ Accommodation of the JT distortions through a relaxation of strain in the presence ${ }^{7}$ or absence ${ }^{8}$ of a disorder at the $A$ site is known to influence the transport properties including metal-insulator transition (MIT) temperature and CMR. ${ }^{7}$ Consequently, the phase coexistence and the percolative mechanism of the MIT and CMR (Ref. 9) are also associated with the structural distortions.

The nature of the charge modulation and its relationship to the structure and other order parameters is a topic of continuous debate. ${ }^{1,3}$ Recently, it was shown that the JT distortions can periodically modulate resulting in a charge-orbital density wave. ${ }^{3,10,11}$ In this Brief Report, we show stripes resembling charge-density wave originating from the intrinsic lattice strain on a La-Ca cation-ordered, rhombohedral superstructure $\mathrm{La}_{0.75} \mathrm{Ca}_{0.25} \mathrm{MnO}_{3}$ (LCMO) film grown strain-free on $\mathrm{MgO}(100)$ substrate.

Epitaxial LCMO film with a La and Ca ordering was grown by metal-organic aerosol deposition technique. ${ }^{12}$ Scanning tunneling microscopy (STM) and scanning tunneling spectroscopy (STS) were performed at a base pressure of $\sim(1-5) \times 10^{-10}$ torr. The film was aligned such that one of the crystallographic axes matched the fast scanning direction-from left to right in all micrographs. The film was loaded into the vacuum chamber after it was cleaned with isopropanol solution. Mechanically cut Pt-Ir tips were used to get high-resolution images in the constant current mode $(I=0.1-0.3 \mathrm{nA})$ with a tip bias ranging from 0.35 to $0.7 \mathrm{~V}$. At room and low temperatures, high-resolution images, within an area of $50 \times 50 \AA^{2}$, were obtained on terraces of $\sim 500-2000 \AA$ length scales. ${ }^{12}$ High-resolution STM at $115 \mathrm{~K}$ is shown in Figs. 1 (a) and 1(b), respectively. One can clearly see the stripelike features with the orientation perpendicular to the scanned direction even in the ferromagnetic metallic state $\left(T_{C} \approx T_{\mathrm{MI}}=273 \mathrm{~K}\right)$, with a predominant peak- to-peak modulation of $6.5-8.5 \AA$. Stripes in the metallic state are counterintuitive because a delocalized $e_{g}$ electron quenches JT distortion and ensues screening of the charge. The room-temperature STM images also revealed stripelike features [Figs. $1(\mathrm{c})$ and $1(\mathrm{~d})$ ], oriented diagonal to the crystallographic axes, with a predominant periodicity of $\sim 5.8 \AA$. These stripes show two different quasiperiodic contgations: a large [black arrows in Fig. 1(e)] corrugation with a width (spread) of $6 \AA$, quite in contrast to the smaller corrugation [orange arrows in Fig. 1(c)], which is $4 \AA$ wide, with a very short-range $(\sim 15 \AA)$ correlation between these features [Fig. 1(d)]. It must be noted that the low-temperature stripes are not directly related to the room-temperature stripes due to the thermal drift associated with disengaging and reengaging of the tip but are related to the same terrace. Since stripe features were also observed in other regions on the film and at different temperatures, we consider it to be representative of the temperattre effect. Further, tip-sample interactions ate evident from the high-resolution micrographs, which could be responsible for pronounced corrugations [Fig. 1(a)] and instability of these stripes.

Criteria for obtaining the atomic resolution by tunneling experiments in manganites were recently expounded based on the reduced screening of the charge due to defect-induced confinement-a trapped polaron. ${ }^{13}$ In hole-doped manganites, it is well known from x-ray scattering experiments that the number of such quasiparticles detectable, in general, is very small. ${ }^{14}$ This further augments the difficulties in obtaining atomic resolution by tunneling experiments on manganites. ${ }^{13,15,16}$ Apart from point defects ${ }^{14}$ and a strong JT-induced charge localization, ${ }^{15,16}$ strain effects have great influence on order parameters and are illustrated to yield $\mathrm{cx}$ otic new phases in manganites. ${ }^{17,18}$ Atomic scale theory considering lattice deformations, indeed, postulates coupled electronic and elastic textures. ${ }^{8}$ In doped manganites, the structural distortions (JT type) are accommodated, and the JT-induced strain is annealed out by cooperative tilting and rotations of octahedra. However, a lack of such cooperativity, locally, perhaps around defects, reduces the screening of the charge within nanometer regions, enhancing the possibility of atomic resolution.

Polarization of the charge leading to stripes in manganites 

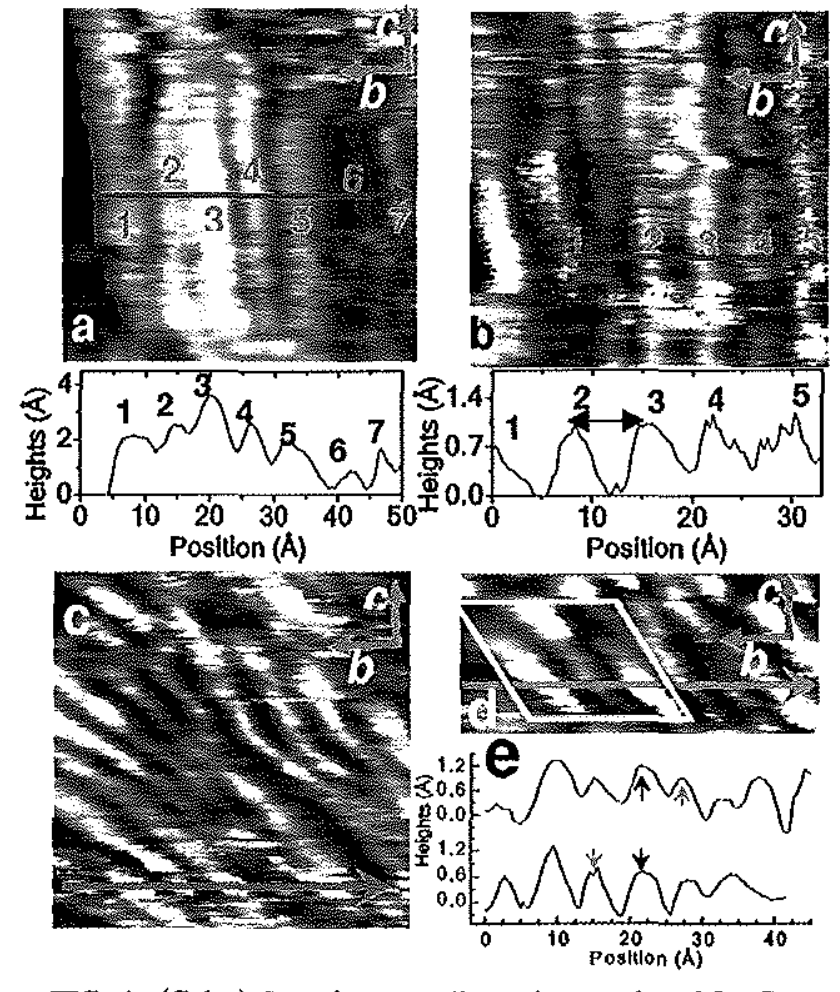

FIG. 1. (Color) Scanning tunneling micrographs of La-Ca ordered $\mathrm{La}_{0.75} \mathrm{Ca}_{0.25} \mathrm{MnO}_{3}$ film. (a) $49.5 \times 49.5 \AA^{2}$ and (b) 50 $\times 50 \AA^{2}$ stripe images obtained at $115 \mathrm{~K}$; the peak-to-peak (black arrows) distance was $\sim 6.5-8.5 \AA$. [(c) and (d)] Room-temperature (294 K) STM images of $45 \times 45$ and $45 \times 20 \AA^{2}$, respectively. (e) Line profiles corresponding to (c) and (d). There are two distinct features as indicated by black and orange arrows. The peak-to-peak distance is $\sim 5.8 \AA$. The two in-plane crystallographic orientations are denoted by arrows at the top left conner in all micrographs.

was shown to be induced by the strain field that determines the nature of overlapping electron wave functionsquadrupolar character: ${ }^{19}$ The direction of the stripe formation was realized by the term $C_{11}-C_{12}-C_{44}$ (where $C_{i j}$ is the elastic modui). A diagonal charge-orbital modulation was associated with the values less than zero, while for values greater than zero, bond-type charge-orbital modulations were suggested; the cross over for optimally doped manganites appears to be related to MIT. ${ }^{20}$ Clearly, the shear $\left(C_{44}\right)$ and uniaxial $\left(C_{11}\right)$ deformations play a role in the stripe formation. Apart from JT-type distortions, the distortions of the $\mathrm{Mn}-\mathrm{O}$ network are also affected by the atomic displacements caused by the ordering of the dissimilar La and Ca cations. In the LCMO film, checkered-board arrangement of uniaxially dilated $\mathrm{La}$ and compressed $\mathrm{Ca}$ unit cells can be deciphered from the cross-sectional high-resolution transmission electron microscopy (HRTEM) images. ${ }^{12}$ The unique ordering generates uniaxially strained $\left(e_{1}\right)$, shear $\left(e_{2}\right)$, and "shuffe"

( $s_{x}$ and $s_{y}$ ) deformations [Fig. 2(a)], as suggested in Ref. 8. It is therefore evident that the cation order is an important parameter in determining the direction and the periodicities of the stripes.

For $T<T_{C}, 6.5-8.5 \AA$ stripe periodicities, with near com-

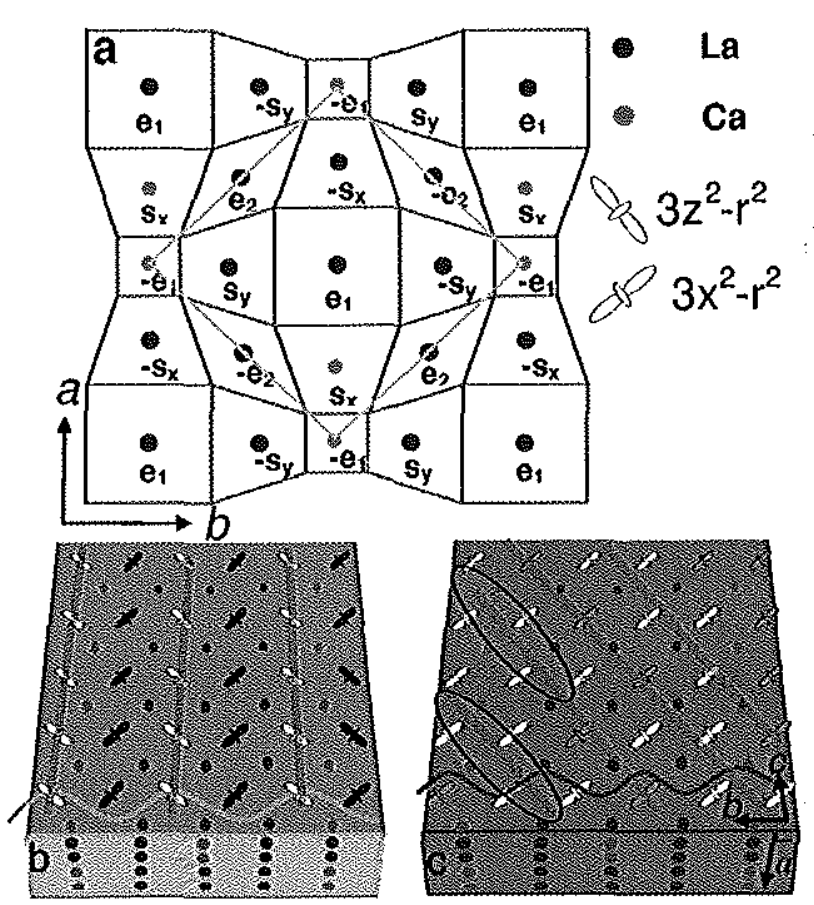

FIG. 2. (Color) (a) Schematic representation of the unit-cell deformations in the LCMO film. The Mn displacements are deduced from the cross-sectional ( $a-b$ plane) HRTEM (Ref. 12). The unitcell lattice deformations are classified as long $\left(e_{1}\right.$ and $\left.e_{2}\right)$ and short range $\left(s_{x}\right.$ and $s_{y}$ ) (Ref. 8). Strain-free superstructure in the $a-b$ plane is shown by the orange square. (b) Schematic representation of bond stripes. $\mathrm{Mn}$ ion in the $b-c$ plane is represented by $d$ orbitals. The lighter colored orbital represents larger distortion than the darker colored. The green lines represent the direction of the charge-orbital stripe and the nonlinear lines denote local density-ofstates (LDOS) modulation across the stripe feature. (c) Probable schematic representation of diagonal stripes within a few nanometer length scale. Two different LDOS features predominantly observed within diagonal stripes are represented by light and dark colored orbitals. "Dimers" of light colored orbitals symbolize clustering (black ovals) of structural distortion that is observed as large and bright features in Figs. 1(c) and 1(d).

mensuration of the charge and the lattice, agree well with the alternating $\mathrm{La}, \mathrm{La} / \mathrm{Ca}$ cation ordering in the $b-c$ plane [Figs. 2(a) and 2(b)]. Taking into account the orientation of the $\mathrm{Mn}$ lattice, from the HRTEM measurements, ${ }^{12}$ the lattice-strainmediated theory discussed above provides rationale for the charge-orbital density-wave features observed along the bond direction [Fig. 2(b)]. The appearance of charge stripes at room temperature with two different corrugations (big and small) and without any apparent lattice, as shown in Figs. 1 (c) and 1(d), could also be related to the weak charge-lattice coupling. Accounting incommensurate periodicity of $\sim 5.8 \AA$ is nontrivial, and an explanation necessitates structural distortions involving two $\mathrm{Mn}$ octahedra-a Peierls-type distortion-as shown in Fig. 2(c). ${ }^{10,11,21,22}$ Such Peierls-type distortions plausibly explain the larger and brighter features within the diagonal stripes.

Given that these stripes in the LCMO film result from a quasiorganization of structural distortions directed by longrange cation ordering, STS offers a better understanding of 


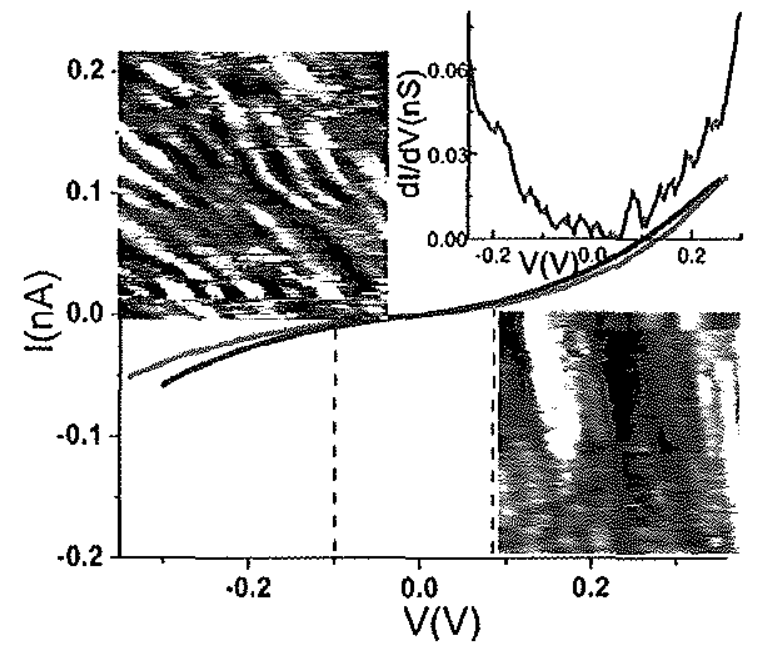

FIG. 3. (Color) Current-voltage (I-V) characteristics of LCMO film. A comparison of $I-V$ data averaged over 12 curves (red) obtained on bond stripes at $115 \mathrm{~K}$ (area of $50 \times 50 \AA^{2}$; bottom-right corner) with an average of $>10^{4}$ data sets corresponding to an area of $500 \times 500 \mathrm{~nm}^{2}$ (blue) at the same temperature. The broken vertical lines indicate the linear part of the $I-V$ curve. Top-right inset: Room-temperature $d I / d V$ spectrum of diagonal stripes averaged over four $I-V$ curves obtained on the stripe area of $45 \times 45 \AA^{2}$ (topleft corner).

the intricacy of competing charge and orbital orders within the nanoscale stripes. The difference in the screening of the charge carricr reflects in the tumneling conductance of the stripe phase. The tumneling current-voltage characteristic (Fig. 3) on the bond stripes reveals a metallic behavior (red curve) distinct from the diagonal stripes, which appear insulatorlike (green curve). Therefore, the room-temperature diagonal stripes, with a depletion $\left(d I / d V_{V \rightarrow 0} \approx 0\right)$ near the Fermi level (inset of Fig. 3), are associated with greater structural distortions. In the bond stripe, the linear part of the $I-V$ curve, which mirrors the "Drude" part to the conductivity, ${ }^{15}$ appears to be less than $0.2 \mathrm{~V}$. Hence, the onset of nonlinear $I-V$ characteristics at lower voltages could be due to a dominant polaronic nature of the conductance. The $I-V$ curve of the bond stripes (red curve) and that averaged over a relatively larger area of $500 \times 500 \mathrm{~nm}^{2}$, where stripes were observed, showed similar characteristics. This implies that the charge fluctuation within the stripes ${ }^{23}$ is similar to that in the bulk, ruling out electronic phase separation with two different conductivities. Besides, metallicity and bond stripe entail charge delocalization and orbital ordering. ${ }^{19,6}$

The fact that the observed stripes are a consequence of quadrupolar characteristics related to the strain can be additionally supported by' second-harmonic generation (SHG) experiments. ${ }^{24}$ In Fig. 4, the SHG field $E_{S H G}(T) \sim I_{S H G}{ }^{1 / 2}$, ( $I_{S H G}$ is the intensity of SHG signal) in the $p$-p geometry ${ }^{24}$ correlates to the quadrate of the magnetization (open circles), $M^{2}(T)$, of the LCMO film, obtained by superconducting

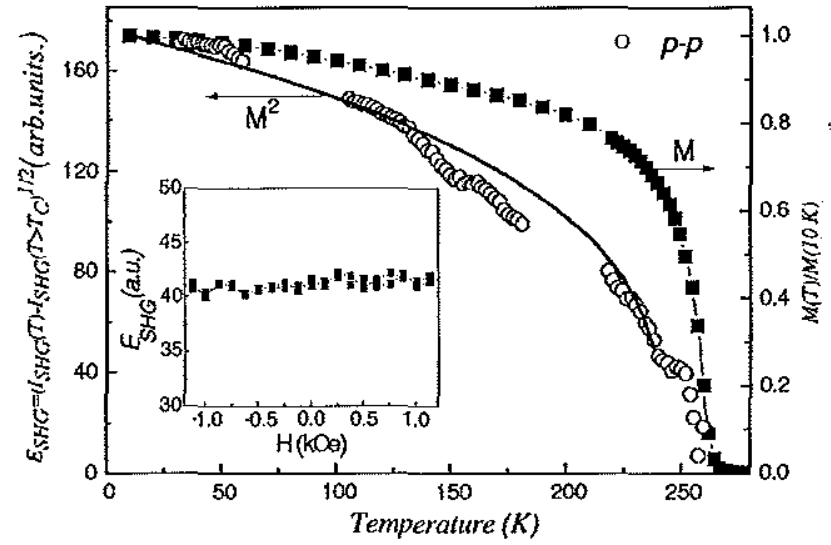

FIG. 4. Temperature dependence of the low-field magnetization $M$ (right scale), measured by SQUID, and the second-harmonic generation (SHG) field $E_{S H G}$ on LCMO film measured in the $p-p$ geometry (Ref. 24). The inset shows magnetic-field dependences of the SHG field $E_{S H G}$ measured at $40 \mathrm{~K}$ for the same polarization combination.

quantum interference device (SQUID) measurements. ${ }^{12}$ However, it should be noted that the discrepancy between the fit and the actual SHG data near $T_{C}$ is due to the changes in the optical constants of the substrate and not related to the sample. ${ }^{24}$ Thus, the quadratic dependence of the $E_{S H G}(T)$ and almost no magnetic-field dependence, $E_{S H G}(M)$, in the $p-p$ geometry (inset Fig. 4) evidence the dominance of the quadrupole contribution. The observation of a strong quadrupolar effect by SHG experiments strengthens the argument proposed for stripe features. Thus, the combined STM and SHG experiments imply a possible orbital ordering within the ferromagnetic phase likely due to the cation order. As regard to stripes, it can be understood to arise from the local strain; which modulates the structural distortions and thereby the orbital. The electron itinerancy in the limit of weak chargelattice coupling could be due to reduced dimensionality of charge-orbital stripe structure. ${ }^{10,11,25}$ Thus, the observed charge-density modulation below $T_{c}$ can be labeled as ferroquadrupolar (orbital) stripes. Further, the nature of the structural distortion, with it the orbital ordering, might be undergoing a change above $T_{c}$ due to the change associated with the strain component. ${ }^{24,26}$ Therefore, these strain-induced stripes provide rationale for the complex structure-property relationship brought out by the propensity of manganites toward new phases such as a polaron liquidlike correlation and metallicity. 25,6

The authors are grateful to P. B. Littlewood for helpful suggestions. They also thank $O$. Shapoval and A. Belenchuk for the help rendered in sample preparation and U. Waghmare, S. A. Koester, and K. Gehrke, for discussions. The work was supported by the Deutsche Forschungsgemeinschaft via SFB 602, project A2. L.S. acknowledges Alexander von Humboldt foundation. 
$\star$ Authors to whom correspondence should be addressed.

tElectronic address: 1sudhee@gwdg.de

łElectronic address: ksamwer@gwdg.de

${ }^{1}$ C. H. Chen and S.-W. Cheong, Phys. Rev. Lett. 76, 4042 (1996).

${ }^{2}$ C. H. Chen, S.-W. Cheong, and A. S. Cooper, Phys. Rev. Lett. 71, 2461 (1993).

${ }^{3}$ J. C. Loudon, S. Cox, A. J. Willians, J. P. Attfield, P. B. Littlewood, P. A. Midgley, and N. D. Mathur, Phys. Rev. Lett. 94, 097202 (2005).

${ }^{4}$ R. von Helmolt, J. Wecker, B. Holzapfel, L. Schultz, and K. Samwer, Phys. Rev. Lett. 71, 2331 (1993).

${ }^{5}$ A. J. Millis, Nature (London) 392, 147 (1998).

${ }^{6}$ R. Kilian and G. Khaliullin, Phys. Rev. B 60, 13458 (1999).

${ }^{7}$ L. M. Rodriguez-Martinez and J. P. Attfield, Phys. Rev. B 58, 2426 (1998).

${ }^{8}$ K. H. Ahn, T. Lookman, and A. R. Bishop, Nature (London) 428 , 401 (2004).

${ }^{9}$ E. Dagotto, Nanoscale Phase Separation and Colossal Magnetoresistance, Springer Series in Solid State Science Vol. 136 (Springer, Berlin, Germany, 2002).

${ }^{10}$ Y.-D. Chuang, A. D. Gromko, D. S. Dessau, T. Kimura, and Y. Tokura, Science 292, 1509 (2001).

It B. J. Campbell, R. Osborn, D. N. Argyriou, L. Vasiliu-Doloc, J. F. Mitchell, S. K. Sinha, U. Ruett, C. D. Ling, Z. Islam, and J. W. Lynn, Phys. Rev. B 65, 014427 (2001).

${ }^{12}$ V. Moshnyaga, L. Sudheendra, O. 1. Lebedev, S. A. Koster, K. Gehrke, O. Shapoval, A. Belenchuk, B. Damaschke, G. van Tendeloo, and K. Samwer, Phys. Rev. Lett. 97, 107205 (2006).

${ }^{13}$ H. M. Rønnow, Ch. Renner, G. Aeppli, T. Kimura, and Y. Tokura,
Nature (London) 440, 1025 (2006)

${ }^{14}$ V. Kiryukhin, A. Borissov, J. S. Ahn, Q. Huang, J. W. Lynn, and S.-W. Cheong, Phys. Rev. B 70, 214424 (2004).

${ }^{15}$ Ch. Renner, G. Aeppli, B.-G. Kim, Y.-A. Soh, and S.-W. Cheong, Nature (London) 416, 518 (2002).

${ }^{16}$ J. X. Ma, D. T. Gillaspie, E. W. Plummer, and J. Shen, Phys. Rev. Lett. 95, 237210 (2005)

${ }^{17}$ G. C. Milward, M. J. Calderón, and P. B. Littlewood, Nature (London) 433, 607 (2005).

${ }^{18}$ K. H. Ahn, T. Lookman, A. Saxena, and A. R. Bishop, Phys. Rev. B 71, 212102 (2005).

${ }^{19}$ D. I. Khomskii and K. I. Kugel, Phys. Rev. B 67, 134401 (2003).

${ }^{20}$ Md. Motin Seikh, C. Narayana, L. Sudheendra, A. K. Sood, and C. N. R. Rao, J. Phys.: Condens. Matter 16, 4381 (2004).

${ }^{21}$ D. V. Efremov, J. Van Den Brink, and D. I. Khomskii, Nat. Mater. 3, 853 (2004).

${ }^{22}$ V. Ferrari, M. Towler, and P. B. Littlewood, Phys. Rev. Lett. 91, 227202 (2003)

${ }^{23} \mathrm{Ch}$. Renner, G. Aeppli, and H. M. Rønnow, Mater. Sci. Eng., C 25, 775 (2005).

${ }^{24}$ E. D. Mishina, A. I. Morosov, A. V. Mishina, V. Moshnyaga, L. Sudheendra, K. Samwer, and Th. Rasing, Phys. Rev. B 75, 064401 (2007).

${ }^{25}$ N. Mannella, W. L. Yang, X. J. Zhou, H. Zheng, J. F. Mitchell, J. Zaanen, T. P. Devereaux, N. Nagaosa, Z. Hussain, and Z.-X. Shen, Nature (London) 438, 474 (2005).

${ }^{26}$ Y. Tokunaga, T. Lottermoser, Y. Lee, R. Kumai, M. Uchida, T. Arima, and Y. Tokura, Nat. Mater. 5, 937 (2006). 